\title{
Adaptive Passivity Based Individual Pitch Control for Wind Turbines in the Full Load Region
}

Sørensen, Kim L. ; Galeazzi, Roberto; Odgaard, Peter F.; Niemann, Hans Henrik; Poulsen, Niels Kjølstad

\section{Published in:}

Proceedings of the 2014 American Control Conference

Link to article, DOI:

10.1109/ACC.2014.6858651

Publication date:

2014

Document Version

Early version, also known as pre-print

Link back to DTU Orbit

Citation (APA):

Sørensen, K. L., Galeazzi, R., Odgaard, P. F., Niemann, H. H., \& Poulsen, N. K. (2014). Adaptive Passivity Based Individual Pitch Control for Wind Turbines in the Full Load Region. In Proceedings of the 2014 American Control Conference (pp. 554-559). IEEE. https://doi.org/10.1109/ACC.2014.6858651

\section{General rights}

Copyright and moral rights for the publications made accessible in the public portal are retained by the authors and/or other copyright owners and it is a condition of accessing publications that users recognise and abide by the legal requirements associated with these rights.

- Users may download and print one copy of any publication from the public portal for the purpose of private study or research.

- You may not further distribute the material or use it for any profit-making activity or commercial gain

- You may freely distribute the URL identifying the publication in the public portal 


\title{
Adaptive Passivity Based Individual Pitch Control for Wind Turbines in the Full Load Region
}

\author{
Kim L. Sørensen ${ }^{1,2}$ and Roberto Galeazzi ${ }^{2}$ and Peter F. Odgaard ${ }^{3}$ and Henrik Niemann ${ }^{2}$ and Niels K. Poulsen ${ }^{4}$
}

\begin{abstract}
This paper tackles the problem of power regulation for wind turbines operating in the top region by an adaptive passivity based individual pitch control strategy. An adaptive nonlinear controller that ensures passivity of the mapping aerodynamic torque-regulation error is proposed, where the inclusion of gradient based adaptation laws allows for the on-line compensation of variations in the aerodynamic torque. The closed-loop equilibrium point of the regulation error dynamics is shown to be UGAS (uniformly globally asymptotically stable). Numerical simulations show that the proposed control strategy succeeds in regulating the power output of the wind turbine despite fluctuations of the wind field due to wake and turbulence, without overloading the pitch actuators.
\end{abstract}

\section{INTRODUCTION}

In recent years the research and development of wind energy harvesting systems focused on optimizing different aspects of the wind turbine in order to improve its Cost of Energy (CoE). Increase the performance of the control system is one of the ways to obtain such an enhancement by optimizing the wind turbine controller in a number of ways. The industrial state-of-the-art solutions rely on PID controllers, which depend upon linearized models of the rotor dynamics, and the strong non-linearities introduced by the aerodynamic torque are handled by gain scheduling. Standard approaches to wind turbine control can be found in [12].

Nowadays, the state-of-the-art wind turbine is a three blades variable speed pitch controlled wind turbine that from a control perspective has two control actuations, namely the blade pitch angle and the generator torque. The overall wind turbine controller has two main control actions, leaving out yaw control, subsystem, and auxiliary controls. Each blade is actuated through a pitch servo, which can change the blade deflection such that the aerodynamics of the wind turbine is modified. These three pitch actuators can be operated with different control references, and if so the controller is called an individual pitch controller (IPC); however this is not the standard solution in which all three pitch actuators are fed with the same control reference. This control strategy is called collective pitch control (CPC). The other control

\footnotetext{
${ }^{1}$ Kim L. Sørensen is with the Centre for Autonomous Marine Operations and Systems, Norwegian University of Science and Technology, Trondheim, Norway kim. sorensenditk.ntnu.no

${ }^{2}$ Roberto Galeazzi and Henrik Niemann are with the Department of Electrical Engineering, Technical University of Denmark, Kgs. Lyngby, Denmark $\{r g, h h n\}$ eelektro.dtu.dk

${ }^{3}$ Peter F. Odgaard is with the Department of Electronic Systems, Aalborg University, Aalborg, Denmark pfodes.aau.dk

${ }^{4}$ Niels K. Poulsen is with the Department of Applied Mathematics and Computer Science, Technical University of Denmark, Kgs. Lyngby, Denmark nkpo@dtu. dk
}

actuation is the generator torque that can be commanded by the electronic converters through which the generator is fully or partly connected to the grid.

Wind turbines typically operates in two general modes (not including low and high wind operation and additional switching modes): partial load and full load. In partial load the objective is to maximize the generated power. In this mode the pitch controllers are idle and the blades are kept at the optimal pitch angle, while the rotor speed is controlled by the generator torque. In full load the pitch actuator are used to keep the rotor torque at the nominal value by controlling the rotor speed, while at the same time the generator torque is typically kept at its nominal value.

In the last decade nonlinear control theory has found an interesting test bed in control design for wind turbine systems, which certainly presents inherent challenges due to the complex non-linearities introduced by the aerodynamic torque. The design of variable speed nonlinear controllers has received particular attention, also due to the fact that the nonlinear effects of the aerodynamic torque significantly simplify when the wind turbine operates in the partial load region. Interesting examples can be found in [3], [4], [5], [8], [20], [22], and [24]. However in the full load region the aerodynamic torque lacks of an explicit representation as a function of the tip speed ratio and the pitch angle; this has represented a major challenge for the application of nonlinear control methods such as e.g. feedback linearization, backstepping, etc., that exploit such representation of the nonlinearities to achieve regulation or tracking. Other control strategies as nonlinear model predictive control (e.g. [10], [15], [21]) and gain scheduling ([2] and references therein) have been proposed for the power regulation problem.

Since a wind turbine is an energy generating unit it would be relevant to use passivity based methods to enforce regulation into the system. Passivity based control has been applied for a fixed pitch passive stall wind turbine [18] with the objective to optimize power production using the generator torque reference as control signal. This study showed an interesting potential for using these methods in wind turbine control. However an active pitch wind turbine is somewhat different, so it may not be obvious how to extend the existing work to this kind of wind turbines, and as well taking into account full load control, where the pitch actuators are active.

This paper addresses the problem of power regulation in the full load region by means of a nonlinear individual pitch controller. Under the assumption of constant generator torque, the control of the generated power is set up as regulation of the rotor angular velocity about its nominal 
value despite wind field fluctuations by means of the blades' pitch angles. First, feedback passivation [14] is applied to establish a passive mapping between the aerodynamic torque and the rotor speed's regulation error. Then damping injection is achieved through proportional feedback. Last, a simple control allocation strategy is applied to distribute the demanded control effort to the individual pitch controllers. The lack of an explicit formulation of the power coefficient is overcome by enhancing the controller through gradient based adaptation laws, which estimates the controller coefficients online. Simulation results confirm the effectiveness of the proposed control scheme, which fulfills the control requirements without overloading the pitch servos.

The theoretical tools utilized, namely feedback passivation and gradient based adaptation laws, are certainly well established; however the novelty of the paper resides in the combination of these tool to provide an effective nonlinear control architecture which achieves power output regulation in the full load region assuming no prior knowledge of the aerodynamic torque.

The paper is organized as follows. Section II presents the wind turbine dynamical model along with the wind field model. Section III is a thorough account of the passivitybased control design procedure, including online adaptation. Section IV presents the simulation results and the performance assessment of the proposed controller. Last, the concluding remarks found in Section $\mathrm{V}$ finalize the paper.

\section{WIND TURBINE MODEL}

The considered wind turbine is a horizontal axis threebladed variable speed variable pitch system, which consists of a wind turbine and a generator. In all aspects of modeling the 3-bladed NREL 5MW offshore baseline wind turbine is utilized [13].

\section{A. Wind field model}

The wind field depends on numerous factors, such as geographical position, season, terrain layout, tower dimensions, etc. The model presented here is an approximation of the wind field that impacts the wind turbine.

The wind field is modeled as wind speed velocity at a given position in the rotor field. The model consists of four different contributions: mean wind $v_{\text {me }}$; wind shear $v_{\mathrm{sh}}$; tower shadow $v_{\text {to }}$; wake $v_{\text {wa }}$. The unified wind field model $v_{w}$ is given by

$$
\begin{aligned}
v_{w}(t ; \Delta r, \theta)= & v_{\text {me }}(t)+v_{\mathrm{sh}}(t ; \Delta r, \theta) \\
& +v_{\text {to }}(t ; \Delta r, \theta)+v_{\text {wa }}(t ; \Delta r, \theta),
\end{aligned}
$$

where $\Delta r$ is the radial distance from the rotor center to any point on the length of the blade, and $\theta$ is the azimuthal angle (or angular coordinate). Wind shear and tower shadow were modeled as in [6], therefore descriptive expressions for those two contributions are omitted for brevity. The wake and stochastic additions have been modeled as in [7], with the exception that in this paper the intensity of the wake has the shape of a Gaussian distribution. For a complete mathematical description of the complete wind field the

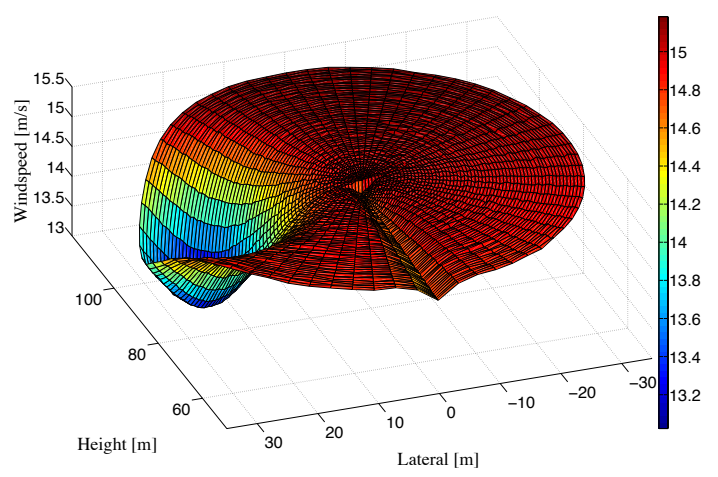

Fig. 1. Complete wind field at a specific time instant.

interested reader is referred to [25]. The wind field at a specific time instant is shown in Fig. 1.

It is assumed that the wind field can be split into three equivalent wind signals, each of which acts on one of the three blades, i.e.

$$
v_{w, b_{j+1}}\left(t ; \theta_{0}+\frac{2 \pi j}{3}\right)=\frac{1}{n} \sum_{i=1}^{n} v_{w, i}\left(t ; \Delta r_{i}, \theta_{0}+\frac{2 \pi j}{3}\right),
$$

with $i$ and $n$ denoting the blade number and total number of blades, respectively; where $j=0,1,2$, and $\theta=\theta_{0}$ represents the azimuthal coordinate associated with the highest point in the rotor field.

\section{B. Turbine model}

A conventional way of characterizing the aerodynamic efficiency of a wind turbine is the power coefficient $C_{P}(\lambda, \beta)^{1}$, which is a function of the tip-speed-ratio $\lambda$ and the pitch angle $\beta . C_{P}$ is given by the ratio between the aerodynamic power $P_{r}$ captured by the wind turbine and the power available $P_{a}$ in the wind area $A$ swept by the blades, i.e.

$$
C_{P}(\lambda, \beta)=\frac{P_{r}}{P_{a}} .
$$

The available power is given by

$$
P_{a}=\frac{1}{2} \rho A v_{w}^{3},
$$

with $\rho$ being the air density, $v_{w}$ denoting the wind speed, and $A=\pi r^{2}$, where $r$ is the blade tip radius. Rearranging (3) and inserting (4) the aerodynamic power is obtained as

$$
P_{r}=\frac{1}{2} \rho A C_{P}(\lambda, \beta) v_{w}^{3},
$$

with $\lambda \triangleq r \omega / v_{w}$, where $\omega$ is the rotor angular velocity. The shape of the function $C_{P}$ depends on the geometry of the wind turbine system. An illustration of a typical $C_{P}$ surface obtained through experimental measurements ([9]) is shown in Fig. 2.

Applying Newton's second law for rotation the wind turbine dynamics is described by

$$
J_{r} \dot{\omega}=T_{a}\left(\lambda, \beta, v_{w}\right)-N_{g} T_{g}
$$

\footnotetext{
${ }^{1}$ The arguments of $C_{P}$ will be hereafter omitted for simplicity
} 


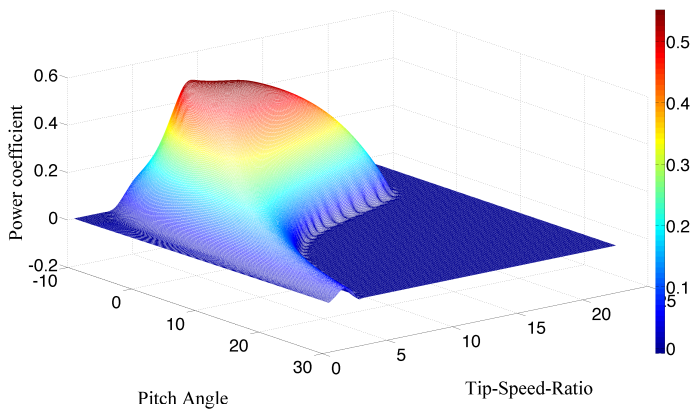

Fig. 2. Power coefficient surface for a typical wind turbine system.

where $J_{r}$ and $N_{g}$ are positive constants denoting the rotor inertia and gear ratio, respectively. $T_{g}$ is the generator torque that is assumed to be constant, whereas the aerodynamic torque $T_{a}$ readily follows from (5) and the definition of $\lambda$

$$
T_{a}=\frac{P_{r}}{\omega}=\frac{1}{2} \rho A r C_{P}(\lambda, \beta) \frac{v_{w}^{2}}{\lambda} .
$$

Equation (6) with (7) is the state equation, where $\beta$ is the controllable input and $\omega$ is controlled output.

\section{NONLINEAR ADAPTIVE INDIVIDUAL PITCH CONTROLLER}

The control objective is to operate the wind turbine in the full load region, ensuring nominal power output $P^{\star}$ despite wind field fluctuations. This objective is achieved through the design of an adaptive nonlinear control law, which first establishes a passive mapping from the aerodynamic torque to the rotor speed's regulation error through feedback passivation [14, Chapter 14], and then ensures uniform global asymptotic stability (UGAS) of the origin of the error dynamics via damping injection.

Due to the lack of an explicit formulation of the aerodynamic torque $T_{a}$, which is only available through the lookup table of the power coefficient $C_{P}$, feedback passivation is achieved by including gradient based adaptation laws that estimate on-line the controller coefficients.

\section{A. Assumptions}

The control law was developed under the following assumptions:

A1 The rotor speed $\omega$ is measured.

A2 Three independent wind speed measurements $v_{w, i}$ are available, one for each blade.

A3 The wind speed is a finite energy, bounded signal, i.e. $v_{w}(t) \in \mathscr{L}_{2} \cap \mathscr{L}_{\infty}$, and $v_{w}(t)>0 \forall t \geq 0$.

A4 The generator torque is kept constant at its nominal value, i.e. $T_{g}=T_{g}^{\star}$.

Concerning assumption A1, measuring $\omega$ is standard practice in windmill systems. Moreover, knowledge of $v_{w, i}$ may be available through wind speed estimators such as those described in [23], or alternatively through LIDAR profiling. Assumption A4 follows from the control objective of constant nominal power output, achieved through set-point regulation of the rotor velocity, i.e.

$$
P=\omega N_{g} T_{g}
$$

\section{B. Controller Design}

The design of the individual pitch controller is based on a time-varying, linear in the parameters approximation of the power coefficient

$$
C_{P}(\lambda, \beta) \approx \breve{C}_{P}(\lambda, \beta)=\alpha_{0}(t)+\alpha_{1}(t) \lambda+\alpha_{2}(t) \beta,
$$

where $\alpha_{j}(t), j=0, \ldots, 2$, are unknown time-varying parameters $^{2}$, whose rate-of-change is assumed to be small, i.e. $\dot{\alpha}_{j} \approx 0$.

Substituting (9) into the aerodynamic torque (7), yields

$$
T_{a}=\frac{1}{2} \rho A r \sum_{i=1}^{n} \frac{1}{n \lambda_{i}} \breve{C}_{P, i}\left(\lambda_{i}, \beta_{i}\right) v_{w, i}^{2},
$$

where the separation of the aerodynamic torque into three equally weighted contributions follows the method suggested in [19]. The system dynamics (6) then reads

$$
\dot{\omega}=\frac{\Phi}{J_{r}}\left(\sum_{i=1}^{n} \frac{1}{n \lambda_{i}} \breve{C}_{P, i}\left(\lambda_{i}, \beta_{i}\right) v_{w, i}^{2}-\frac{N_{g}}{\Phi} T_{g}^{\star}\right),
$$

where $\Phi=\rho A r / 2$, and the output measurement vector is $\mathbf{y}=\left[\omega, v_{w, 1}, v_{w, 2}, v_{w, 3}\right]^{\mathrm{T}}$.

The main contribution of the paper is the following.

Proposition 1: Consider the regulation error dynamics

$$
\dot{e}_{\omega}=-\frac{\Phi}{J_{r}}\left(\sum_{i=1}^{n} \frac{1}{n \lambda_{i}} \breve{C}_{P, i}\left(\lambda_{i}, \beta_{i}\right) v_{w, i}^{2}-\frac{N_{g}}{\Phi} T_{g}^{\star}\right),
$$

where $e_{\omega}(t)=\omega_{d}-\omega(t)$ is the regulation error, and $\omega_{d}$ is the constant nominal set-point for the rotor speed. Then, the individual pitch control law $(i=1, \ldots, N)$

$$
\beta_{i}=\frac{1}{\hat{\alpha}_{2, i}}\left[-\hat{\alpha}_{0, i}-\hat{\alpha}_{1, i} \lambda_{i}+\frac{\lambda_{i}}{v_{w, i}^{2}}\left(\frac{N_{g}}{\Phi n} T_{g}^{\star}-u_{C, i}\right)\right],
$$

where $u_{C, i}$ is an additional control component to be chosen, together with the adaptation laws

$$
\begin{aligned}
& \dot{\hat{\alpha}}_{0, i}=-\Gamma_{i} \frac{v_{w, i}^{2} e_{\omega}}{\lambda_{i}}, \\
& \dot{\hat{\alpha}}_{1, i}=-\Gamma_{i} v_{w, i}^{2} e_{\omega}, \\
& \dot{\hat{\alpha}}_{2, i}=-\Gamma_{i} \operatorname{Proj}\left(\frac{v_{w, i}^{2} e_{\omega} \beta_{i}}{\lambda_{i}}\right),
\end{aligned}
$$

render the origin of (12) UGAS.

Remark 2: The operator $\operatorname{Proj}(\cdot)$ is the projection operator [16, Lemma E.1], which is used to guarantee a bounded away from zero estimate of the uncertain input gain for each pitch control signal.

Proof: Consider the radially unbounded and positive definite function Lyapunov function candidate

$$
V\left(e_{\omega}, \tilde{\alpha}_{p, i}\right)=\frac{1}{2}\left(\frac{J_{r}}{\Phi} e_{\omega}^{2}+\sum_{i=1}^{n} \frac{1}{n \Gamma_{i}} \tilde{\alpha}_{p, i}^{2}\right)
$$

\footnotetext{
${ }^{2}$ The time argument will hereafter be omitted for simplicity.
} 
with $p=0,1,2$ denoting the parameter number, $\Gamma_{i} \in \mathbb{R}_{+}$the adaptation rate for the individual pitch controller. The time derivative of $V$ along the trajectories of the system reads

$$
\begin{aligned}
\dot{V}= & \frac{J_{r}}{\Phi} e_{\omega} \dot{e}_{\omega}+\sum_{i=1}^{n} \frac{1}{n \Gamma_{i}} \tilde{\alpha}_{p, i} \dot{\tilde{\alpha}}_{p, i} \\
= & -e_{\omega}\left(\sum_{i=1}^{n} \frac{v_{w, i}^{2}}{n \lambda_{i}}\left(\alpha_{0, i}+\alpha_{1, i} \lambda_{i}+\tilde{\alpha}_{2, i} \beta_{i}+\hat{\alpha}_{2, i} \beta_{i}\right)-\frac{N_{g}}{\Phi n} T_{g}^{\star}\right) \\
& -\sum_{i=1}^{n} \frac{1}{n \Gamma_{i}}\left(\tilde{\alpha}_{0, i} \dot{\hat{\alpha}}_{0, i}+\tilde{\alpha}_{1, i} \dot{\hat{\alpha}}_{1, i}+\tilde{\alpha}_{2, i} \dot{\hat{\alpha}}_{2, i}\right),
\end{aligned}
$$

where the dynamics of the estimation errors are given by $\dot{\tilde{\alpha}}_{p, i}=-\dot{\hat{\alpha}}_{p, i}\left(\right.$ since $\left.\dot{\alpha}_{p, i} \approx 0\right)$, with the estimation errors defined as $\tilde{\alpha}_{p, i}=\alpha_{p, i}-\hat{\alpha}_{p, i}$.

Inserting the control law (13) into (18) yields

$$
\begin{aligned}
\dot{V}= & -e_{\omega} \sum_{i=1}^{n} \frac{v_{w, i}^{2}}{n \lambda_{i}}\left(\tilde{\alpha}_{0, i}+\tilde{\alpha}_{1, i} \lambda+\tilde{\alpha}_{2, i} \beta_{i}\right)+e \sum_{i=1}^{n} \frac{1}{n} u_{C, i} \\
& -\sum_{i=1}^{n} \frac{1}{n \Gamma_{i}}\left(\tilde{\alpha}_{0, i} \dot{\hat{\alpha}}_{0, i}+\tilde{\alpha}_{1, i} \dot{\hat{\alpha}}_{1, i}+\tilde{\alpha}_{2, i} \dot{\hat{\alpha}}_{2, i}\right) .
\end{aligned}
$$

Rearranging (19) as

$$
\begin{aligned}
\dot{V}= & -\frac{1}{n} \sum_{i=1}^{n}\left[\tilde{\alpha}_{0, i}\left(\frac{e_{\omega} v_{w, i}^{2}}{\lambda_{i}}+\frac{\dot{\hat{\alpha}}_{0, i}}{\Gamma_{i}}\right)+\tilde{\alpha}_{1, i}\left(e_{\omega} v_{w, i}^{2}+\frac{\dot{\hat{\alpha}}_{1, i}}{\Gamma_{i}}\right)\right. \\
& \left.+\tilde{\alpha}_{2, i}\left(\frac{e_{\omega} v_{w, i}^{2} \beta_{i}}{\lambda_{i}}+\frac{\dot{\hat{\alpha}}_{2, i}}{\Gamma_{i}}\right)-e_{\omega} u_{C, i}\right]
\end{aligned}
$$

and inserting the adaptation laws (14)-(16), results in

$$
\dot{V}=e_{\omega} \sum_{i=1}^{n} \frac{1}{n} u_{C, i}
$$

Defining the output as $y=e_{\omega}$, the system with input $u=$ $\frac{1}{2 n} \rho A r \sum_{i=1}^{n} u_{C, i}$ and output $y$ is passive with the storage function $V$. Moreover, the system is zero-state observable. Setting $u_{C, i} \triangleq-\kappa_{d, i} e_{\omega}$ with $\kappa_{d, i}>0$, the equilibrium point of (12), (14)-(16) is concluded to be uniformly stable, i.e. $e_{\omega}$ and $\tilde{\alpha}_{p, i}$ are bounded. Further, since $e_{\omega}=\omega_{d}-\omega$, and $\omega_{d}$ is constant, then $\omega$ is bounded. To show that the regulation error converges asymptotically to zero the second derivative of $V$ is calculated

$$
\ddot{V}=-2 e_{\omega} \dot{e}_{\omega} \sum_{i=1}^{n} \frac{1}{n} \kappa_{d, i}
$$

The closed loop error dynamics is obtained by inserting (13) into (12)

$$
\dot{e}_{\omega}=-\frac{\Phi}{J_{r} n} \sum_{i=1}^{n}\left[\frac{v_{w, i}^{2}}{\lambda_{i}}\left(\tilde{\alpha}_{0, i}+\tilde{\alpha}_{1, i} \lambda_{i}+\tilde{\alpha}_{2, i} \beta_{i}\right)-\kappa_{d, i} e_{\omega}\right]
$$

which shows that $\dot{e}_{\omega}$ is uniformly bounded (note that the signals $v_{w, i}$ are bounded according to assumption A3), hence $\ddot{V}$ is bounded, implying that $\dot{V}$ is uniformly continuous. Application of Barbalat's lemma [14] proves that $e_{\omega} \rightarrow 0$ as $t \rightarrow \infty$, therefore, $\omega$ uniformly asymptotically converges to $\omega_{d}$.
TABLE I

Parameter Values at Operating Point.

\begin{tabular}{cccccc}
\hline $\bar{v}_{\mathrm{me}}[\mathrm{m} / \mathrm{s}]$ & $\bar{\omega}[\mathrm{rad} / \mathrm{s}]$ & $\bar{\lambda}[-]$ & $\bar{\beta}\left[^{\circ}\right]$ & $\bar{C}_{P, d}[-]$ & $\bar{T}_{g}[\mathrm{kNm}]$ \\
\hline 15 & 1.2671 & 5.4485 & 11.0064 & 0.1814 & 40.6806 \\
\hline
\end{tabular}

Remark 3: The control input

$$
u=\frac{1}{2 n} \rho A r \sum_{i=1}^{n} u_{C, i}
$$

is the total aerodynamic torque that is needed in order to achieve regulation of $e_{\omega}$. Hence setting the control signals to $u_{C, i} \triangleq-\kappa_{d, i} e_{\omega}$ can be seen as a simple control allocation strategy, that defines which share of the total aerodynamic torque $u$ is delivered by each pitch controller. Moreover, the proportional feedback $u_{C, i}$ are injecting damping into the system.

\section{PERFORMANCE ASSESSMENT}

\section{A. Operating point}

The system is designed to operate in the full load region characterizzed by wind speeds above $11.4 \mathrm{~m} / \mathrm{s}$ for this particular wind turbine [13]. To initialize the nonlinear simulation model at an operating point the following procedure is followed. First a mean wind speed $\bar{v}_{\text {me }}$ is selected, then the rotor speed $\bar{\omega}$ is obtained from [13], and the value of the tip-speed-ratio $\bar{\lambda}$ follows from its definition. The generator torque $\bar{T}_{g}$ is obtained by

$$
\bar{T}_{g}=T_{g}^{\star}=\frac{P^{\star}}{N_{g} \bar{\omega}},
$$

where $P^{\star}=5 \mathrm{MW} . \bar{C}_{P, d}$ is determined by

$$
\bar{C}_{P, d}=\frac{P^{\star}}{P_{a}}
$$

where $P_{a}$ is found from (4). Last, the pitch angle $\bar{\beta}$ is determined through simulations.

Note that to obtain $\bar{\beta}$ the initial estimates of $\hat{\alpha}_{0, i}, \hat{\alpha}_{1, i}$, and $\hat{\alpha}_{2, i}$ have been determined through simulation. The mean wind speed selected for simulations reflects an initial condition related to a configuration for nominal power generation. The parameters obtained are found in Table I.

\section{B. Simulation Results}

To assess the asymptotic regulation capabilities of the designed adaptive passivity-based individual pitch controller, the system is tested on a deterministic simulation environment where only step changes in the mean wind $v_{\text {me }}(t)$ are considered. Subsequently, the controller is tested on a stochastic simulation environment, by applying the wind field model presented in Section II-A, for the purpose of evaluating the performance under more realistic operational conditions.

The responses to the deterministic simulation, driven by a low-pass filtered square wave wind signal centered around 

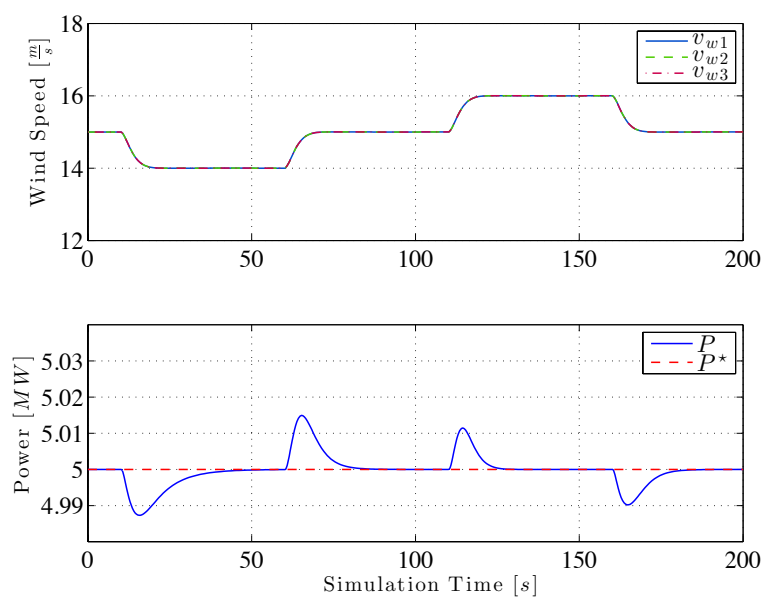

Fig. 3. Rotor speed regulation in deterministic simulation environment1. Wind field signal for each blade, 2. Nominal power and power output response.

$15 \mathrm{~m} / \mathrm{s}$ and with an amplitude of $\pm 1 \mathrm{~m} / \mathrm{s}$, is shown in Figs. 34. Clearly, the proposed control scheme achieves smooth and precise asymptotic rotor speed regulation, thereby maintaining power output at its nominal value, despite the complete lack of knowledge of the aerodynamic torque.

The stochastic simulation is driven by the wind field presented in Section II-A, and the responses are displayed in Figs. 5-7. The closed loop system shows good set-point regulation capabilities of the desired rotor speed with a mean squared regulation error of $3.37 \cdot 10^{-7} \mathrm{rad}^{2} / \mathrm{s}^{2}$, and it fulfills the control objective with a standard deviation for the power output of $2.29 \mathrm{~kW}$. It is also worth noting that the control objective is fulfilled with a control effort in line with the system capabilities with a maximum pitch rate of $1.18 \%$, which is considerable lower than the pitch rate saturation limit of $\pm 10^{\circ} / \mathrm{s}$, hence the control scheme could be applicable for larger scale wind turbines with slower pitch actuators. The pitch rates are displayed in the center plot of fig. 6 . Finally, the parameter estimates display similar behavior for each blade, and as seen in Figure 7 assumes values in close proximity to their initial guesses.

\section{CONCLUSION}

Maximizing power output while alleviating structural stress for wind turbines operating in the full load region is essential to prolong wind turbine longevity and decrease operation and maintenance costs. This paper has proposed a nonlinear adaptive passivity-based individual pitch control strategy for the control of a variable speed variable pitch wind turbine operating in the full load region. Due to the lack of an explicit analytical expression for the nonlinear power coefficient, the designed controller was augmented with gradient based adaptation laws to estimate the controller coefficients online.

It was shown that the proposed controller guarantees uniform global asymptotic stability of the rotor speed's regulation error. Deterministic and stochastic simulations
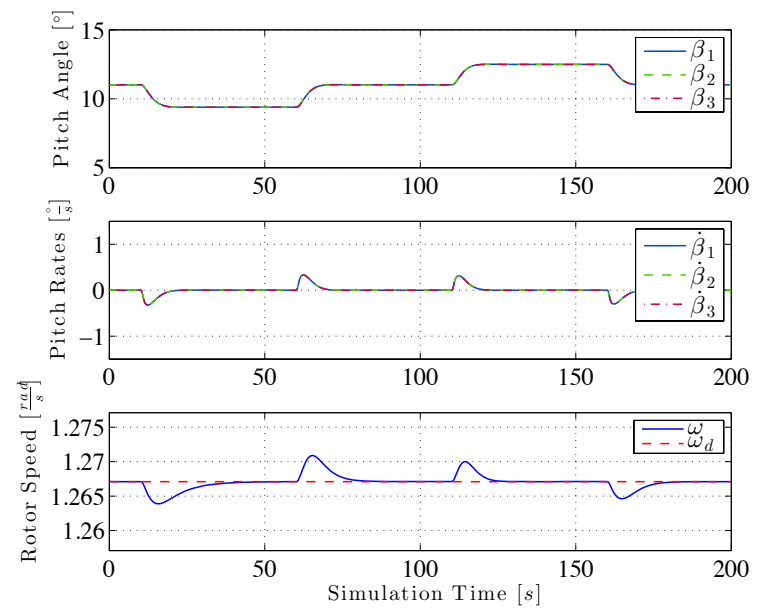

Fig. 4. Rotor speed regulation in deterministic simulation environment- 1. Pitch signals for each blade, 2. Pitch rate signals for each blade, 3. Desired and actual rotor speed.
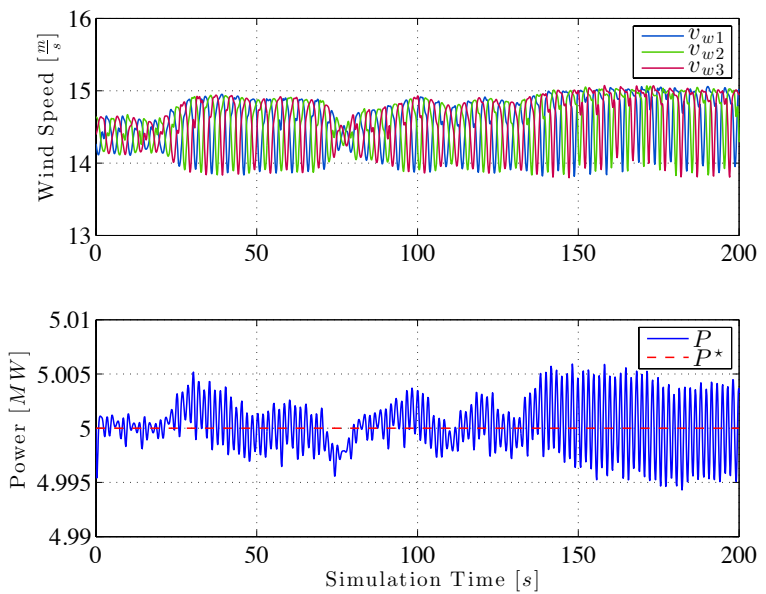

Fig. 5. Rotor speed regulation in stochastic simulation environment- 1 Wind field signal for each blade, 2. Nominal power and power output response.

confirmed the expected performance: despite model uncertainties and a largely fluctuating wind field the rotor speed is promptly and successfully regulated to its nominal value, which in turn guarantees the nominal electrical power output. Further the control objective was fulfilled with a control effort well within the capabilities of the system.

\section{REFERENCES}

[1] B. Beltram, T. Ahmed-Ali, and M. El Hachemi Benbouzid. Sliding mode power control of variable-speed wind energy conversion systems. IEEE Transactions on Energy Conversion, 23:551-558, 2008.

[2] F. D. Bianchi, H. De Batista, and R. J. Mantz. Wind Turbine Control Systems - Principles, Modeling and Gain Scheduling Design. Springer, 2007.

[3] B. Boukhezzar and H. Siguerdidjane. Nonlinear control of variable speed wind turbines without wind speed measurement. In Proceedings of the 44th IEEE Conference on Decision and Control, 2005.

[4] B. Boukhezzar and H. Siguerdidjane. Nonlinear control of a variablespeed wind turbine using a two-mass model. IEEE Transactions on Energy Conversion, 26(1):149-162, March 2011. 

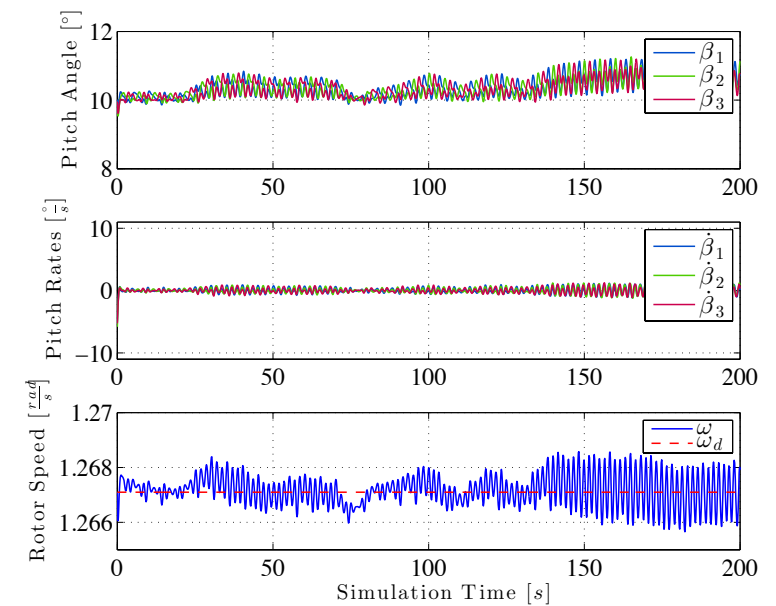

Fig. 6. Rotor speed regulation in stochastic simulation environment- 1 . Pitch signals for each blade, 2. Pitch rate signals for each blade, 3. Desired and actual rotor speed.

[5] R. Burkart, K. Margellos, and J. Lygeros. Nonlinear control of wind turbines: An approach based on switched linear systems and feedback linearization. In Proceedings of the 50th IEEE Conference on Decision and Control and European Control Conference, pages 5485-5490, Orlando, Fl, December 2011.

[6] D. S. L. Dolan and P. W. Lehn. Simulation model of wind turbine $3 \mathrm{p}$ torque oscillations due to wind shear and tower shadow. IEEE Transactions on Energy Conversion, 21(3):717-724, September 2006.

[7] J. Friis, E. Nielsen, J. Bonding, F. D. Adegas, J. Stoustrup, and P. F. Odgaard. Repetitive model predictive approach to individual pitch control of wind turbines. In Proceedings of the 50th IEEE Conference on Decision and Control and European Control Conference, pages 3664-2670, 2011.

[8] R. Galeazzi, M. P. S. Gryning, and M. Blanke. Observer backstepping control of variable speed wind turbine. In Proceedings of the 2013 American Control Conference, 2013.

[9] L. C. Henriksen. Model predictive control of a wind turbine. Master's thesis, Technical University of Denmark, 2007.

[10] L. C. Henriksen, N. K. Poulsen, and M. H. Hansen. Nonlinear Model Predictive Control of a Simplified Wind Turbine, pages 551556. International Federation of Automatic Control, 2011.

[11] E. Iyasere, M. H. Salah, D. M. Dawson, J. R. Wagner, and E. Tatlicioglu. Robust nonlinear control strategy to maximize energy capture in a variable speed wind turbine with an internal induction generator. Journal of Control Theory and Applications, 10(2):184-194, 2012.

[12] K.E. Johnson, M.J.. Pao, L.Y.and Balas, and L.J. Fingersh. Control of variable-speed wind turbines - standard and adaptive techniques for maximizing energy capture. IEEE Control Systems Magazine, 26(3):71-81, June 2006

[13] J. Jonkman, S. Butterfield, W. Musial, and G Scott. Definition of a 5MW reference wind turbine for offshore system development. Offshore wind, National Renewable Energy Laboratory, 2009.

[14] H. K. Khalil. Nonlinear Systems. Prentice Hall, 3rd edition, 2002.

[15] A. Koerber and R. King. Nonlinear model predictive control for wind turbines. In Proceedings of EWEA, Bruxelles, Belgium, March 2011. EWEA, EWEA.
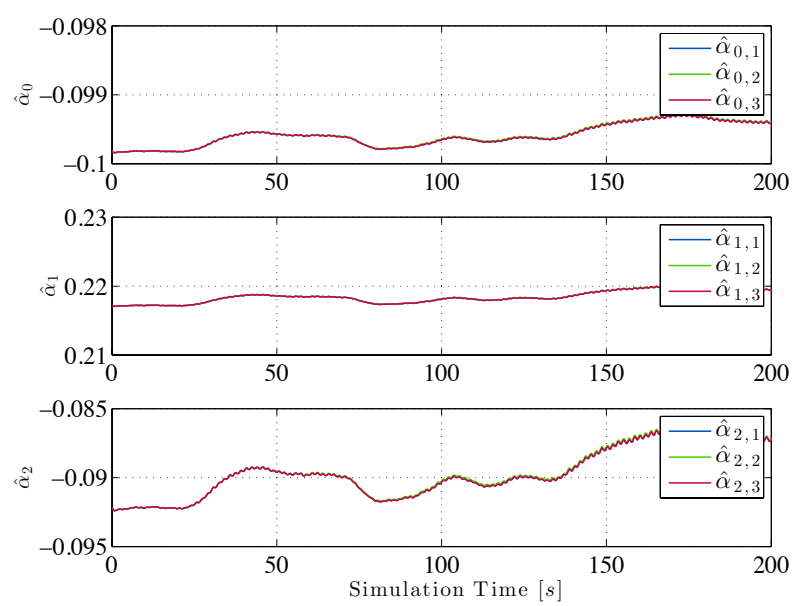

Fig. 7. Rotor speed regulation in stochastic simulation environment- 1 . Pitch signal for each blade, 2-4. Parameter estimates for adaptive control laws.

[16] M. Krstic, I. Kanellakopoulos, and P. Kokotovic. Nonlinear and Adaptive Control Design. Wiley Interscience, 1995.

[17] Z. Lu and W. Lin. Asymptotic tracking control of variable-speed wind turbines. In Proceedings of the 18th IFAC World Congress, volume 18th, 2011.

[18] F. Mancilla-David and R. Ortega. Adaptive passivity-based control for maximum power extraction of stand-alone windmill systems. Control Engineering Practice, 20(2):173-181, February 2012.

[19] P. F. Odgaard, J. Stoustrup, and M. Kinnaert. Fault-tolerant control of wind turbines: A benchmark model. IEEE Transactions on Control Systems Technology, 21(4):1168-1182, 2013.

[20] U. Ozbay, E. Zergeroglu, and S. Sivrioglu. Adaptive backstepping control of variable speed wind turbines. International Journal of Control, 81:6:910-919, 2011.

[21] D. Schlipf, D. J. Schlipf, and M. Kühn. Nonlinear model predictive control of wind turbines using lidar. Wind Energy, n/a:n/a, 2012.

[22] S. Sivrioglu, U. Ozbaky, and E. Zerge. Variable speed control of wind turbines: A robust backstepping approach. In Proceedings of the 17th IFAC World Congress, 2008.

[23] M.N. Soltani, T. Knudsen, M. Svenstrup, R. Wisniewski, P. Brath, R. Ortega, and K. Johnson. Estimation of rotor effective wind speed: A comparison. IEEE Transactions on Control Systems Technology, 21(4):1155-1167, 2013.

[24] Y.D. Song, B. Dhinakaran, and X.Y. Bao. Variable speed control of wind turbines using nonlinear and adaptive algorithms. Journal of Wind Engineering and Industrial Aerodynamics, 85:293-308, 2000.

[25] K. L. Sørensen. Nonlinear repetitive control of wind turbines. Master's thesis, The Technical University of Denmark (DTU), Automation and Control (AUT)., May 2013.

[26] O. Wasynczuk, D. T. Man, and J. P. Sullivan. Dynamic behavior of a class of wind turbine generators during random wind fluctuations. IEEE Transactions on Power Apparatus and Systems, 100(6):2838$2845,1981$. 